\title{
Erratum to: Socio-demographic Moderators of Associations Between Psychological Factors and Latinas' Breast Cancer Screening Behaviors
}

\author{
L. G. Perez ${ }^{1,2}$ - J. P. Elder ${ }^{2,3}$ - J. Haughton ${ }^{2}$ M. E. Martinez ${ }^{4,5}$ - E. M. Arredondo ${ }^{2,3}$
}

Published online: 9 August 2017

(C) Springer Science+Business Media, LLC 2017

\section{Erratum to: J Immigrant Minority Health DOI 10.1007/s10903-017-0633-1}

The original version of this article unfortunately contained a mistake in "Perceived barriers to breast cancer screening" subsection. The sentence "Responses ranged from $1=$ "strongly agree" to $5=$ "strongly disagree"." should read as "Responses ranged from $1=$ "strongly disagree" to $5=$ "strongly agree"." The corrected text is given below:
Nine items from the 1990 Tampa survey [21] were used to assess perceived barriers to breast cancer screening (e.g., "getting a mammogram is a frightening experience"). Responses ranged from 1 = "strongly disagree" to $5=$ "strongly agree". Responses were averaged with higher mean scores indicating higher perceived barriers to screening. The sample Cronbach's alpha $=0.82$.

The online version of the original article can be found under doi:10.1007/s10903-017-0633-1.

L. G. Perez

lgperez12@gmail.com

1 Joint Doctoral Program in Public Health, University of California, San Diego/San Diego State University, San Diego, CA, USA

2 Institute for Behavioral and Community Health, San Diego, CA, USA

3 Division of Health Promotion and Behavioral Science, San Diego State University, San Diego, CA, USA

4 Moores Cancer Center, University of California, San Diego, La Jolla, CA, USA

5 Department of Family Medicine and Public Health, University of California, San Diego, La Jolla, CA, USA 